\title{
Breathers on lattices with long range interaction
}

\author{
S. Flach \\ Max-Planck-Institute for the Physics of Complex Systems, Nöthnitzer Str. 38, D-01187 Dresden, Germany
}

(August 13, 2018)

\begin{abstract}
We analyze the properties of breathers (time periodic spatially localized solutions) on chains in the presence of algebraically decaying interactions $1 / r^{s}$. We find that the spatial decay of a breather shows a crossover from exponential (short distances) to algebraic (large distances) decay. We calculate the crossover distance as a function of $s$ and the energy of the breather. Next we show that the results on energy thresholds obtained for short range interactions remain valid for $s>3$ and that for $s<3$ (anomalous dispersion at the band edge) nonzero thresholds occur for cases where the short range interaction system would yield zero threshold values.

03.20.+i, 03.65.-w, 03.65.Sq
\end{abstract}

The understanding of dynamical localization in classical spatially extended and ordered systems experienced recent considerable progress. Specifically time-periodic and spatially localized solutions of the classical equations of motion exist, which are called (discrete) breathers, or intrinsic localized modes. The attribute discrete stands for the discreteness of the system, i.e. instead of field equations one typically considers the dynamics of degrees of freedom ordered on a spatial lattice. The lattice Hamiltonian is invariant under discrete translations in space. The discreteness of the system produces a cutoff in the wavelength of extended states, and thus yields a finite upper bound on the spectrum of eigenfrequencies $\Omega_{q}$ (phonon band) of small-amplitude plane waves (we assume that usually for small amplitudes the Hamiltonian is in leading order a quadratic form of the degrees of freedom). If now the equations of motion contain nonlinear terms, the nonlinearity will in general allow to tune frequencies of periodic orbits outside of the phonon band, and if all multiples of a given frequency are outside the phonon band too, there seems to be no further barrier preventing spatial localization (for reviews see [1], [2]).

To cope with breathers in lattice dynamics, one has to face the problem of (i) quantization of breathers, ii) breathers in the presence of acoustic phonon bands and iii) breathers in the presence of long range interactions (e.g. in ionic crystals). While (i) still lacks a full understanding, the correspondence between classical breathers and quantum bound states is believed to be correct ( [3], 近, [5], 6, [7]). The case of acoustic breathers has been studied in one dimension in [8] and in two dimensions in [9], where it was shown that the resonance of a zero frequency component of the breather (static deformation) with the zero of the acoustic spectrum leads to an algebraically decaying lattice deformation, but not to a disappearance of the breather.

As for the case of long range interactions, less results are known (but see e.g. [10], [12]). A general proof of existence of breathers in $d$-dimensional lattices with algebraically decaying interactions was obtained in [11] with upper bounds for the spatial decay of the breather amplitude. Namely for interactions decaying like $1 / r^{s}$ with $r$ being the distance from the breather center and $s$ some power $s>d$, the breather amplitude is bounded from above by a power law $a / r^{s}$ with $a$ being some nonzero constant This result leaves us with two questions. First, how can one obtain contact with the case of short range interaction (basically $s \rightarrow \infty$ ) where exponential localization takes place? And secondly, what is happening to energy thresholds of discrete breathers in the presence of long range interactions? In the case of short range interaction, simple estimates of the far distance energy of a breather solution yield the correct predictions for nonzero energy thresholds [14]. Obviously these estimates would yield zero energy thresholds for all cases of long range interactions if the far distance energy is calculated with the help of a spatial decay $a / r^{s}$ for the breather (the far distance energy would be simply $\sim a^{2}$, and would always tend to zero if $a \rightarrow 0$, see also [13]). We will resolve these puzzles in the following.

At this stage it is appropriate to fix the class of Hamiltonians to be considered further:

$$
H=\sum_{l}\left[\frac{1}{2} P_{l}^{2}+V\left(X_{l}\right)+\sum_{l^{\prime}} W_{\left|l-l^{\prime}\right|}\left(X_{l}-X_{l^{\prime}}\right)\right] .
$$

Here $P_{l}$ and $X_{l}$ are canonically conjugated scalar momenta and displacements of a particle at lattice site $l$. The on site potential $V(z)=\sum_{\mu=2}^{\infty} \frac{v_{\mu}}{\mu} z^{\mu}$ can be used as a simple way to generate an optical phonon spectrum, and the interaction $W_{l}(z)=\sum_{\mu=2}^{\infty} \frac{\phi_{\mu}(l)}{\mu} z^{\mu}$ should incorporate longe range interactions with $\phi_{2}(l)=\frac{C}{2} \frac{1}{l^{s}}$. For small values of $P_{l}$ and $X_{l}$ the classical Hamiltonian equations of motion $\dot{X}_{l}=\frac{\partial H}{\partial P_{l}}, \dot{P}_{l}=-\frac{\partial H}{\partial X_{l}}$ can be linearized in $X_{l}$. The corresponding eigenvalue problem when solved for plane waves $X_{l}(t) \sim \exp ^{\mathrm{i}\left(q l-\Omega_{q} t\right)}$ is given by

\footnotetext{
1 This is in contrast to results obtained in 12 and 13 , where for $d=1$ and $s>3$ exponential decay was obtained. Actually both decay laws hold, see below.
} 


$$
\Omega_{q}^{2}=v_{2}+2 C \sum_{m=1}^{\infty} \frac{1}{m^{s}}(1-\cos (q m))
$$

Let us discuss the properties of $E_{s}(q)=\Omega_{q}^{2} \geq 0$. First $E_{s}(q)$ is bounded from above for all $s>1$ and periodic in $q$ with period $2 \pi$. Most important is that $E_{s}(q)$ is a nonanalytic function in $q$, i.e. its $\kappa=(s-1)$-st derivative with respect to $q$ is discontinuous at $q=0$ (when $s$ is noninteger, $(s-1)<\kappa<s)$. This follows already from the fact that the convergence radius of (2) is zero for nonzero imaginary components in $q$. Indeed for even integers $s$ one finds 15] $\left(E_{s}(q)-v_{2}\right) \sim B_{s}(q /(2 \pi))$ for $0 \leq q \leq 2 \pi$. Here $B_{s}(z)$ is the Bernoulli polynomial of $s$-th order. Consequently at small $q$ the expansion of $E_{s}(q)$ contains a term $q^{s-1}$ which leads together with the periodicity of $E_{s}(q)$ to the mentioned nonanalyticity. For odd integers $s$ the expansion of $E_{s}(q)$ contains a term $q^{s-1} \ln (q)$, and for noninteger $s$ a term $q^{s-1}$ (follows from $\mathrm{d}^{2} E_{s}(q) / \mathrm{d} q^{2}=-E_{s-2}(q)+2 C \zeta(s-2)$ with $\zeta(z)$ being the Riemann Zeta function). Finally for small $\mathrm{q}$ the leading term in the expansion of $E_{s}(q)$ is $v_{2}+C \zeta(s-2) q^{2}$ for $s>3$ and $v_{2}+2 C a(s) q^{s-1}$ for $1<s<3$ with $a(s)=\int_{0}^{\infty}(1-\cos x) / x^{s} \mathrm{~d} x[13]$. Note that the dispersion at the upper band edge $(q=\pi)$ is completely analytical, and in leading order always proportional to $(q-\pi)^{2}$. Some of these results have been discussed at length in [16] (see also original references therein).

Now we can turn to the first problem of the spatial decay of a breather. In order to generate a breather solution we chose $v_{4} \neq 0$ and all other anharmonic terms in $V(z)$ and $W(z)$ being zero. Since we can only simulate finite system sizes $N$, we use periodic boundary conditions. In that case we have to define a cutoff length in the interaction which we chose to be $N / 2$ (we will discuss the corresponding corrections to $E_{s}(q)$ later). We calculate breather solutions using a Newton algorithm (see [17], [2] for details). The results for $s=10,20,30$ are shown in Fig.1.

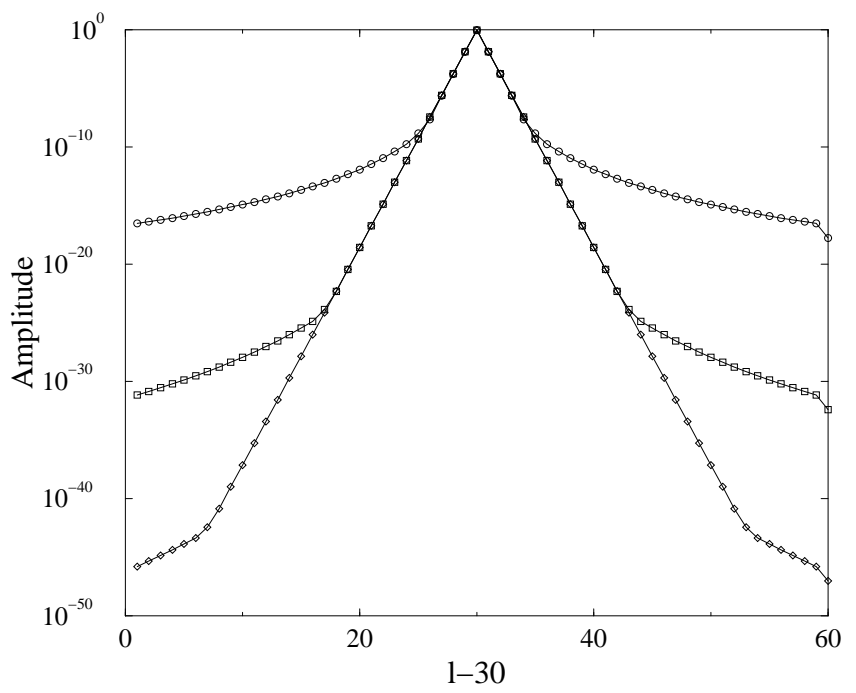

FIG. 1. Breather solution at time $t=0$ with $P_{l}(t=0)=0$. The corresponding displacements (amplitudes) $X_{l}(t=0)$ are plotted versus lattice site. The nonzero model parameters are $v_{2}=v_{4}=1, C=0.01$. The frequency of the solutions is chosen $T=4.7682$. Circles: $s=10$, squares: $s=20$, diamonds: $s=30$. Lines are guides to the eye.

We observe that the spatial decay of the breather is exponential for small distances from the center, while it becomes algebraic (in fact exactly $1 / l^{s}$ ) after a crossover at some distance $l_{c}$ (see Fig.2).

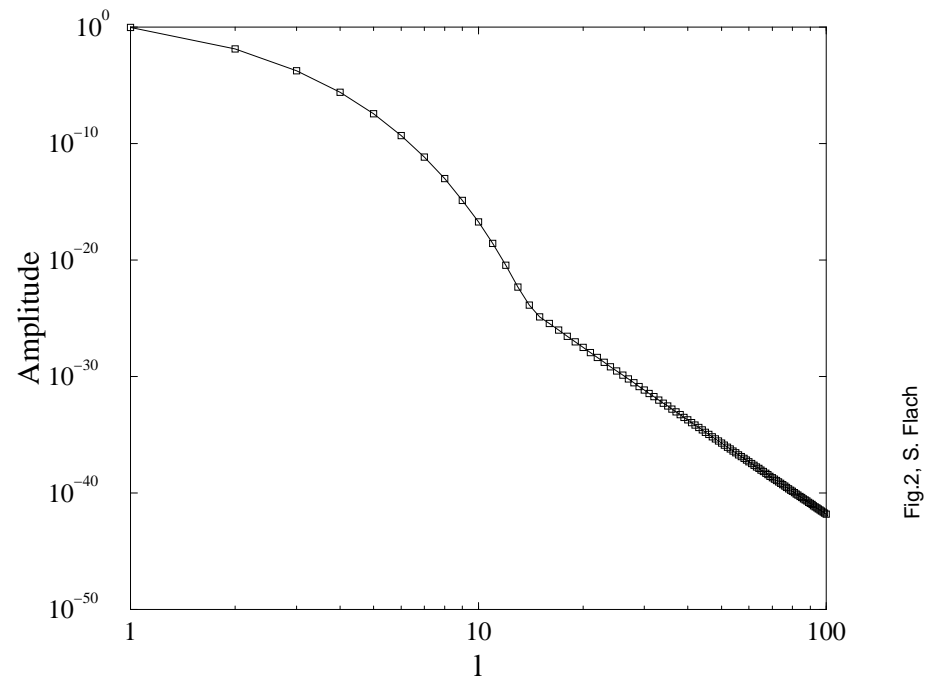

FIG. 2. Same as in Fig.1 but only for $s=30$ in a log-log plot.

Note that $l_{c}$ is $s$-dependent. Moreover, $l_{c}$ is also depending on the parameter which selects a given breather solution from its one-parameter family (this parameter could be the breather frequency, its energy, action or something else). In order to understand this result we can proceed along the following path. Since the breather amplitude decays to zero with increasing distance from the center, we can linearize the equations of motion far from the breather center, keeping the information that we deal with a time-periodic solution with frequency $\omega_{b}$ fulfilling the nonresonance condition $k \omega_{b} \neq \Omega_{q}$ (see e.g. [2] for details and also for exceptional nonlinear corrections which are however not important at this stage). Assuming $X_{l}(t)=\sum_{k} x_{l}(k) \mathrm{e}^{\mathrm{i} k \omega_{b} t}$ we find linear difference equations for $x_{l}(k)$ which do not mix in $k$-space. The spatial decay of the $k$-th amplitude is then given by 등

$$
G_{\lambda}(l)=\int_{0}^{2 \pi} \frac{\cos (q l)}{\Omega_{q}^{2}-\lambda} \mathrm{d} q, \quad \lambda=k^{2} \omega_{b}^{2} .
$$

The spatial decay of the breather is thus given by the convergence properties of the Fourier series, whose coefficients are given by the rhs in (3). As is known, nonanalytic functions with discontinuities in the $(s-1)$-st derivative (cf. the integrand on the rhs in (30) produce Fourier 
series which converge algebraically $1 / l^{s}$ 15. From that follows that at large distances the spatial decay of the breather will be algebraic, which is what we found in Fig.1. To obtain the exponential decay at small distances, let us first slide along the breather family such that the breather frequency (or one of its multiples) approaches the edge of the phonon band $\Omega_{q}$. Then the integrand (3) will become very large for wave numbers close to the band edge which is approached. Applying a stationary phase approximation to (3), i.e. expanding the integrand around the band edge we obtain

$$
G_{\lambda}(l) \sim \int_{-\infty}^{\infty} \frac{\cos (q l)}{v_{2}-\lambda+C \zeta(s-2) q^{2}} \mathrm{~d} q
$$

for $s>3$ and

$$
G_{\lambda}(l) \sim \int_{0}^{\infty} \frac{\cos (q l)}{v_{2}-\lambda+2 C a(s) q^{s-1}} \mathrm{~d} q
$$

for $1<s<3$. Standard evaluation of (何) (closing the integration contour in the complex plane by adding a half circle with infinite radius and evaluating the residua) yields $G_{\lambda}(l) \sim \mathrm{e}^{-\sqrt{v_{2}-\lambda l}}$ for $s>3$, i.e. exponential decay [12,13]! On the other side, (5) yields (closing the integration contour in the complex plane by adding a quarter circle and returning to zero along the positive imaginary axis, and noticing that there are no poles of the integrand in the enclosed first quadrant including the imaginary axis) $G_{\lambda}(l) \sim 1 / l^{s}$ for $1<s<3$ 12, 13.

Now we can explain the observed crossover from exponential to algebraic decay in Fig.1. Indeed, the stationary phase approximation for these cases leads to (画) in the limit $\left(v_{2}-\lambda\right) \rightarrow 0$. This approximation neglects higher order terms in the expansion of $E_{s}(q)$ around $q=0$ which necessarily contain nonanalytic terms. Consequently (4) probes (3) for not too large distances (this is counterintuitive to the assumption that the stationary phase approximation is correct for large $l$ [12,13], which it is not). Thus we can explain the observed crossover. We can even estimate the crossover distance $l_{c}$ using a simple argument. A tagged site with index $l<l_{c}$ and $l>0$ (the center of the breather is located at $l_{b}=0$ ) will experience forces from all other sites with index $l^{\prime}$ according to (1). The amplitude of these forces will monotonously decay to zero for increasing $l^{\prime}$ with $l^{\prime}>l$. However the amplitude of the forces for decreasing $l^{\prime}$ will be given by $\left(l-l^{\prime}\right)^{-s} \mathrm{e}^{\nu\left(\left(l-l^{\prime}\right)\right.}$ for $0<l^{\prime}<l$ (here $\nu$ is the given exponent of the spatial decay for $|l|<l_{c}$ ). Since for negative $l^{\prime}$ the amplitude of these forces will again monotonously decay to zero, the worst case is reached when $l^{\prime}=0$. If this force acting from the center of the breather on site $l$ is comparable to the forces acting on $l$ from its nearest neighbours, the exponential decay will be violated. This condition yields $l_{c}^{-s} \mathrm{e}^{\nu l_{c}}=1$ or

$$
\frac{\ln l_{c}}{l_{c}}=\frac{\nu}{s} .
$$

This equation has either two solutions or none. For the larger (physically relevant) solution we find $l_{c} \rightarrow \infty$ if $\nu / s \rightarrow 0$, while the smaller one yields 1 in this limit and is not of interest. Thus for $s>3$ exponential decay is reobtained either for large $s$ or for breathers with frequencies close to the phonon band edge. Since we are considering a lattice, the exponential decay part will disappear if $l_{c} \approx 1$ or smaller. For $s=20$ and $\nu=4.2724$ we obtain $l_{c}=11.39$, and for $s=30$ and the same value of $\nu$ the result is $l_{c}=21.56$. We miss the observed crossovers in Fig. 1 by just two sites.

For $1<s<3$ no exponential decay is observed provided the breather frequency is located in the gap below the phonon band. For breather frequencies above the phonon band the dispersion at the upper band edge yields always quadratic dependence in $q$ (see above) and thus there will be always a crossover from exponential to algebraic decay (provided $l_{c}>1$ ). All these results were verified by calculating corresponding breather solutions.

To conclude this part we want to stress that a modified interaction $\phi_{2}(l) \sim(-1)^{l} / l^{s}$ will simply exchange the notation of upper and lower phonon band edges, and the case of acoustic interactions is obtained by letting $v_{2} \rightarrow 0$.

Let us now turn to the question of energy thresholds for breathers in the presence of long range interactions. There are two lines of argumentation known from the short range interaction case [14]. The first one estimates the far distance energy of the breather solution in the limit when the amplitude of the breather center is small and thus its frequency is close to a phonon band edge (the only limit where the breather energy can actually become small). Using exponential spatial decay the result is that the breather energy tends to zero only if $v_{3} \neq 0$ or/and $v_{4} \neq 0$, stays finite if $v_{3}=v_{4}=0$ and $v_{5} \neq 0$ or/and $v_{6} \neq 0$, and diverges if $v_{3}=v_{4}=v_{5}=v_{6}=0$ and $v_{\mu} \neq 0$ for some $\mu \geq 7$ (see [14] for details). In the case of long range interaction $l_{c}$ tends to infinity in this limit for $\omega_{b}>\Omega_{q}$ or $\omega_{b}<\Omega_{q}$ and $s \geq 3$. Consequently the breather energy will have the same qualitative behaviour as in the case of short range interaction (the results are similar to those obtained in 14 with the tendency that the height and the position of the energy minima shift to larger values with decreasing values of $s$ ).

However for $\omega_{b}<\Omega_{q}$ and $1<s<3$ no exponential decay is observed and the far distance energy of the breather is given by $\sim A^{2} \int \frac{1}{r^{2 s}} \mathrm{~d}^{d} r$ where $A$ is the amplitude of the breather center. This energy will always vanish in the limit of zero amplitude. However we are in posess of a second line of argumentation for the behaviour of the breather energy at small amplitudes. For that we consider a finite system of $N$ sites. As was shown in [18], the band edge plane waves (BEPW) (which can be rigorously defined in the limit of vanishing amplitudes) undergo tangent bifurcations, which give birth to discrete breathers. The amplitude $A_{c}$ of the BEPW at the 
bifurcation point (for nonvanishing cubic and/or quartic terms in the Hamiltonian) was calculated in [18]:

$$
A_{c} \sim \sqrt{\left|\Omega_{B E P W}^{2}-\Omega_{q_{1}}^{2}\right|}
$$

where $q_{1}$ denotes the wavevector closest to the band edge wavevector. Here we consider periodic boundary conditions and a cutoff in the interaction at one half of the system size. This cutoff will induce finite size corrections to the dispersion $\Omega_{q}^{2}$ for all $q$ except for the band edge points. With $q_{1}=\frac{2 \pi}{N}$ this correction amounts to $\Delta_{q_{1}}=\Omega_{q_{1}}^{2}(\infty)-\Omega_{q_{1}}^{2}(N)=2 C \sum_{m=N / 2+1}^{\infty} \frac{1}{m^{s}}\left(1-\cos \left(\frac{2 \pi}{N} m\right)\right)_{\text {Acknowledgements. }}^{\text {temperatures. }}$

Evaluation of (7) for $s>1$ gives

$$
\frac{\Delta_{q_{1}}}{2 C} \approx b(s)\left(\frac{2 \pi}{N}\right)^{s-1}-2\left(\frac{2}{N}\right)^{s}
$$

with $b(s)=\int_{\pi}^{\infty} \frac{1}{x^{s}}(1-\cos x) \mathrm{d} x$. Consequently the correct result for $(7)^{\pi}$ and $1<s<3$ is $A_{c}^{2} \sim c(s) / N^{s-1}$ with $c(s)=\int_{0}^{\pi} \frac{1}{x^{s}}(1-\cos x) \mathrm{d} x$. The total energy $E_{c} \sim N A_{c}^{2}$ in the bifurcation point for $1<s<3$ is finally given by

$$
E_{c} \sim N^{2-s}
$$

This has to be contrasted to the case of short range interactions in one-dimensional systems which can be obtained from (10) by choosing $s=3$ and is $E_{c} \sim 1 / N$. We thus find, that anomalous dispersion at the band edge $\sim q^{s-1}$ for $1<s<3$ even further supports the divergence of the breather energy at small amplitudes, since for cubic and quartic anharmoncities in the Hamiltonian, for which no divergence in energy is found for short range interactions, energy divergence is obtained for long range interaction with $s<2$. These results confirm studies of nonlinear Schrödinger chains with long range interactions, where $s<2$ marks the appearance of two stable soliton solutions compared to one for $s>2$ 12.

Let us discuss the results. First, we numerically confirm that discrete breathers persist in the case of long range interactions, even in the case of anomalous dispersions at the band edge. Secondly, the spatial decay of breathers is characterized by a crossover length which separates exponential from algebraic decay. Third, we show that the existence of energy thresholds for breather solutions is supported by long range interactions, and can take place when short range interactions (e.g. in onedimensional systems) are not capable of producing these thresholds. Thus we can state, that discrete breathers appear independent of the lattice dimension and survive acoustic and anomalous dispersions. Discrete breathers have energy thresholds provided the lattice dimension is large enough (typically $d \geq 2$ ) or the interaction is long ranged (for $d=1 s<2$ ).

Let us speculate on the value of these energy thresholds for the lattice dynamics of crystals. As there is no small parameter in the system, these threshold energies will be comparable to the energy of a vacancy. Consequently discrete breathers in two- or three-dimensional crystal lattices will be high energy excitations, which could play a role close to the melting transition. In contrast for one-dimensional systems there are no energy thresholds (except for $s<2$ ) and breathers can play a role in the dynamics of molecules and similar objects also at low

I wish to thank D. Bonart and J. B. Page for many discussions which initiated these studies, C. Baesens and R. S. MacKay for sending me their work prior publication (and drawing my attention to the helpful reference [15]) and Yu. Gaididei, M. Katsnelson, Yu. Kosevich and O. Yevtushenko for helpful discussions.

[1] S. Aubry. Physica D, 103:201, 1997.

[2] S. Flach and C. R. Willis. Phys. Rep., 295:181, 1998.

[3] A. A. Ovchinnikov. Zh. Eksp. Teor. Fiz. / Soviet Physics JETP, 57 / 30:263 / 147, 1969 / 1970.

[4] A. A. Ovchinnikov and H. S. Erikhman. Uspekhi Fiz. Nauk (russian), 138:289, 1982.

[5] S. Aubry, S. Flach, K. Kladko, and E. Olbrich. Phys. Rev. Lett., 76:1607, 1996.

[6] S. Flach and V. Fleurov. J. Phys.: Cond. Mat., 9:7039, 1997.

[7] W. Z. Wang, A. R. Bishop, J. T. Gammel, and R. N. Silver. Phys. Rev. Lett., 80:3284, 1998.

[8] R. Livi, M. Spicci, and R. S. MacKay. preprint, 1997.

[9] S. Flach, K. Kladko, and S. Takeno. Phys. Rev. Lett., 79:4838, 1997.

[10] D. Bonart. Physics Letters, A231:201, 1997.

[11] C. Baesens and R. S. MacKay. Preprint, 1998.

[12] Yu. B. Gaididei, S. F. Mingaleev, P. L. Christiansen, and K. O. Rasmussen. Phys. Rev., E55:6141, 1997.

[13] S. Flach. Physica D, 113:184, 1998.

[14] S. Flach, K. Kladko, and R. S. MacKay. Phys. Rev. Lett., 78:1207, 1997.

[15] A. Zygmund. Trigonometric Series. Cambridge University Press, 1968.

[16] A. A. Maradudin, E. W. Montroll, and G. H. Weiss. Theory of Lattice Dynamics in the Harmonic Approximation. Academic Press, New York, 1963.

[17] J. L. Marin and S. Aubry. Nonlinearity, 9:1501, 1996.

[18] S. Flach. Physica, D91:223, 1996. 